\section{SAT0292 EVALUATION OF RADIOGRAPHIC PROGRESSION AFTER 4 YEARS OF ETANERCEPT (ETN) IN ANKYLOSING SPONDYLITIS (AS): RESULTS FROM THE OPEN-LABEL EXTENSION (OLE) OF THE PHASE 3 CLINICAL TRIAL}

N. Haroon ${ }^{1}$, R.D. Inman ${ }^{1}$, M. Fairbairn ${ }^{2} .{ }^{1}$ University Health Network, University of Toronto, Toronto; ${ }^{2}$ Amgen Canada Inc., Mississauga, Ontario, Canada

Background: ETN was well tolerated and showed clinical efficacy (ASAS 20: ETN $59 \%$, placebo $28 \%, p<0.0001$ ) through 24 wks in a phase $3 \mathrm{AS}$ trial: ${ }^{1}$ efficacy was sustained up to 2 years in pts who completed the study and continued ETN in an OLE. ${ }^{2}$ No significant difference was found in change in modified Stoke AS Spine Score (mSASSS) from baseline (BL) to yr 2 of the OLE among ETN-treated pts vs a historic cohort not treated with tumour necrosis factor inhibitors (TNFi). ${ }^{3}$ Objectives: Report radiographic progression through 4 years in ETN-treated pts with AS.

Methods: In the double-blind, placebo-controlled phase 3 study, pts with active AS were randomised to ETN $25 \mathrm{mg}$ BIW or placebo for 24 wks. Pts who completed the study could enrol in a 168-wk OLE and were treated with ETN $25 \mathrm{mg}$ BIW (amended after 17 months to $50 \mathrm{mg} \mathrm{QW}$ ). The primary radiographic endpoint was change in mSASSS from BL to yr 2 vs change in mSASSS from yr 2 to yr 4 . Results: 257 pts were treated in the OLE, of whom $126(49.0 \%)$ completed the study and $131(51.0 \%)$ withdrew prior to 168 wks (most common reasons [ $>5 \%]$ : pt refusal [10.1\%]; adverse event, infection, or injection-site reaction [8.2\%]; lack of efficacy [7.8\%]). ETN resulted in sustained improvement in signs and symptoms of active disease for up to 168 wks beyond the 24-wk double-blind study. Of 267 pts who received $>1$ dose of ETN in the phase 3 study or OLE, 124 were included in the 4 year primary radiographic endpoint analysis ( 8 received a prohibited TNFi and were excluded). BL characteristics were similar between these pts and all pts who received $\geq 1$ dose of ETN in the phase 3 study. Mean change in mSASSS among completers was 1.96 from $B L$ to yr $4(n=110)$ while change in mean mSASSS between yr 2 and yr 4 was $0.66(n=109)$. The nominal $p$-value for change in mSASSS from BL to yr 2 vs change from yr 2 to yr 4 was 0.0536 . Radiographic data suggest disease progression continued in pts receiving ETN continuously over 4 years; however, mean mSASSS increased from BL to year 2 and not from yr 2 to yr 4 (figure 1) due to a few outlier patients with large mSASSS values at yr 2 but missing 4 year data.

Abstract SAT0292 - Table 1. Baseline Demographic and Disease Characteristics Pts Who Received $\geq 1$ Dose of ETN in the Phase 3 Study or Its Extension and Had Baseline, 2 year, and 4 year X-rays $(\mathrm{n}=127)$

\begin{tabular}{lc}
\hline Mean (SD) age, yrs & $42.43(9.63)$ \\
Male, $\mathbf{n}(\%)$ & $89(70.1)$ \\
HLA-B27 positive, $\mathbf{n}(\%)$ & $100(78.7)$ \\
Used NSAIDs, $\mathbf{n}(\%)$ & $115(90.6)$ \\
Used DMARDs, $\mathbf{n}(\%)$ & $43(33.9)$ \\
Used corticosteroids, $\mathbf{n}$ & $16(12.6)$ \\
(\%) & \\
Mean (SD) mSASSS & $16.7(16.3)$ \\
Mean (SD) disease & $10.61(8.67)$ \\
duration, yrs & \\
\hline
\end{tabular}

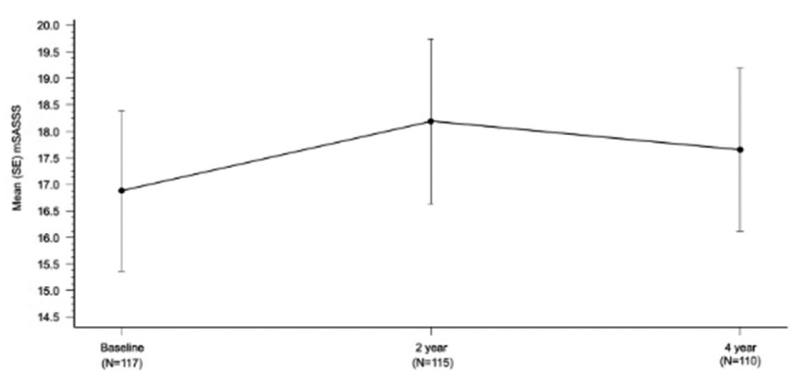

Abstract SAT0292 - Figure 1. Mean mSASSS

Conclusions: This is the first report of 4 year radiographic ETN data in AS, and these data suggest that disease progression continued in pts who received ETN continuously throughout, but that disease progression may be slower after longerterm treatment with ETN vs shorter term. This adds to the already-existing data that demonstrate TNFi seem to reduce radiographic progression in pts with AS.

\section{REFERENCES:}

[1] Davis Arthritis Rheum 2003.

[2] Davis Ann Rheum Dis 2005
[3] van der Heijde Arthritis Rheum 2008.

Acknowledgements: Study supported by Amgen, Inc. Medical writing assis tance provided by BlueMomentum, an Ashfield Company, and funded by Amgen Inc.

Disclosure of Interest: N. Haroon Consultant for: Abbvie, Amgen, Janssen, Merck, Novartis, UCB, R. Inman Consultant for: Amgen, Abbvie, Janssen, Merck, Novartis, M. Fairbairn Shareholder of: Amgen, Employee of: Amgen DOI: 10.1136/annrheumdis-2018-eular.3810

\section{SAT0293 SAFETY AND EFFICACY OF TOFACITINIB, AN ORAL JANUS KINASE INHIBITOR, UP TO 36 MONTHS IN PATIENTS WITH ACTIVE PSORIATIC ARTHRITIS: DATA FROM THE THIRD INTERIM ANALYSIS OF OPAL BALANCE, AN OPEN-LABEL, LONG-TERM EXTENSION STUDY}

P. Nash ${ }^{1}$, L.C. Coates $^{2}$, A.J. Kivitz ${ }^{3}$, P.J. Mease ${ }^{4}$, D.D. Gladman ${ }^{5}$, J.

A. Covarrubias-Cobos ${ }^{6}$, D. Fleishaker ${ }^{7}$, C. Wang ${ }^{7}$, E. Kudlacz ${ }^{7}$, S. Menon ${ }^{7}$,

L. Fallon ${ }^{8}$, T. Hendrikx ${ }^{9}$, K.S. Kanik ${ }^{7} .{ }^{1}$ University of Queensland, Brisbane,

Australia; ${ }^{2}$ University of Oxford, Oxford, UK; ${ }^{3}$ Altoona Center for Clinical Research,

Duncansville; ${ }^{4}$ Swedish Medical Center and University of Washington, Seattle,

USA; ${ }^{5}$ University of Toronto, Toronto Western Hospital, Toronto, Canada; ${ }^{6}$ Unidad

Reumatologica Las Americas S.C.P, Mérida, Mexico; ${ }^{7}$ Pfizer Inc, Groton, USA

${ }^{8}$ Pfizer Canada, Montreal, Canada; ${ }^{9}$ Pfizer Inc, Collegeville, USA

Background: Tofacitinib is an oral Janus kinase inhibitor for the treatment of psoriatic arthritis (PsA)

Objectives: To report the safety, tolerability and efficacy of tofacitinib in patients (pts) with active PsA from an ongoing, open-label, long-term extension (LTE) study (OPAL Balance, NCT01976364; November 2017 data-cut; database not locked).

Methods: Eligible pts from 2 Phase $(\mathrm{P}) 3$ tofacitinib PsA studies (OPAL Broaden, NCT01877668; OPAL Beyond, NCT01882439) entered a 3 year LTE $\leq 3$ months after completing the $\mathrm{P} 3$ study or discontinuing for reasons unrelated to study drug Pts received tofacitinib $5 \mathrm{mg}$ BID to Month (M)1, after which dose adjustments between 5 and $10 \mathrm{mg}$ BID were permitted to improve efficacy, or for safety reasons. Pts receiving a csDMARD at $\mathrm{P} 3$ study entry continued the same csDMARD in the LTE. Primary endpoints were incidence and severity of adverse events (AEs) and changes from baseline $(\Delta)$ in laboratory values. Safety data are reported up to M36. Efficacy was evaluated up to M30 (when $n>50$ ) as a secondary endpoint.

Results: 686 pts were treated in OPAL Balance; $468(68.2 \%)$ remained in the study at data cut-off. Mean (range) LTE tofacitinib exposure was 614 (1-1032 days. On Day 1, 675 pts $(98.4 \%)$ received a csDMARD, which was discontinued in 86 pts (12.7\%). To M36, 2189 AEs were reported in 546 pts $(79.6 \%), 95$ pts $(13.8 \%)$ had serious AEs and 59 pts $(8.6 \%)$ discontinued due to AEs. Serious infections occurred in 12 pts $(1.7 \%)$, herpes zoster $(\mathrm{HZ})$ in 20 pts $(2.9 \%$; 1 serious event), major adverse cardiovascular events in 5 pts $(0.7 \%)$, malignancies in 24 pts (3.5\%; including 12 pts with NMSC) and uveitis in 2 pts $(0.3 \%)$. No AEs of gas trointestinal perforation or inflammatory bowel disease were reported. There were 5 deaths (not attributed to treatment, as assessed by the investigator) due to metastatic pancreatic carcinoma, acute cardiac failure/hypertensive heart disease, chronic obstructive pulmonary disease, pulmonary embolism and cardiovascular insufficiency. Four AEs of latent tuberculosis were reported in pts whose previously negative QuantiFERON response became positive. ALT was elevated $\geq 3 \times$ ULN in 27 pts (4.0\%), and AST $\geq 3 \times$ ULN in 15 pts (2.2\%). Changes in laboratory values observed in P3 studies were generally stable in the LTE, except for a modest decrease in absolute lymphocyte count over time. Eight pts $(1.2 \%)$ discontinued (protocol-mandated) due to laboratory value changes. ACR

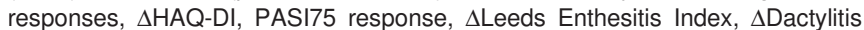
Severity Score and $\triangle$ Pain were maintained up to $M 30$.

Conclusions: Over 36 months in the LTE, the safety profile of tofacitinib in active PsA pts was generally similar to that of the P3 studies. No new safety risks were identified. Efficacy across various PsA disease domains was maintained over time.

Acknowledgements: Study sponsored by Pfizer Inc. Medical writing support was provided by A MacLachlan of CMC and funded by Pfizer Inc.

Disclosure of Interest: P. Nash Grant/research support from: AbbVie, BristolMyers Squibb, Eli Lilly, Janssen, Novartis, Pfizer Inc, Roche, Sanofi, UCB, Consultant for: AbbVie, Bristol-Myers Squibb, Eli Lilly, Janssen, Novartis, Pfizer Inc, Roche, Sanofi, UCB, Speakers bureau: AbbVie, Bristol-Myers Squibb, Eli Lilly, Janssen, Novartis, Pfizer Inc, Roche, Sanofi, UCB, L. Coates Grant/research support from: AbbVie, Janssen, Consultant for: AbbVie, Amgen, Bristol-Myers Squibb, Celgene, Janssen, Eli Lilly, MSD, Novartis, Pfizer Inc, Sun Pharma, UCB, A. Kivitz Consultant for: AbbVie, Celgene, Genentech, Genzyme, Janssen, Merck, Novartis, Pfizer Inc, Sanofi, UCB, Speakers bureau: AbbVie, Celgene, 